\title{
Inflation from Extra Dimensions
}

\author{
Janna J. Levin \\ Canadian Institute for Theoretical Astrophysics \\ Mc Lennan Labs, 60 St. George Street, Toronto, ON M5S 1A7
}

\begin{abstract}
A gravity-driven inflation is shown to arise from a simple higher dimensional universe. In vacuum, the shear of $n>1$ contracting dimensions is able to inflate the remaining three spatial dimensions. Said another way, the expansion of the 3 -volume is accelerated by the contraction of the $n$-volume. Upon dimensional reduction, the theory is equivalent to a four dimensional cosmology with a dynamical Planck mass. A connection can therefore be made to recent examples of inflation powered by a dilaton kinetic energy. Unfortunately, the graceful exit problem encountered in dilaton cosmologies will haunt this cosmology as well.
\end{abstract}

98.80.Hw, 98.80.Cq,04.50.+h

to be published in Phys. Lett B 


\section{I.}

Imagine a spacetime which is created empty. Even in the absence of matter as a source of fuel, the evolution of the spacetime can still involve interesting dynamics. The shearing and twisting of dimensions can feed each other, driving some dimensions to expand and others to contract.

Suppose a universe is created initially with some spatial dimensions contracting and the others expanding. The shear of the contracting dimensions has been shown to expand the other dimensions at a humble pace [1]. The expansion of the spatial sections decelerates with time. It is the intent of this paper to present an additional possibility. The contraction of some dimensions can actually inflate the expanding space; that is, the expansion of the spatial sections can accelerate with time.

Consider a universe with $n$ extra spatial dimensions, in addition to the 3 space and 1 time dimensions we occupy. A general vacuum solution describing $n$ such contracting dimensions and 3 expanding spatial dimensions was found nearly fourteen years ago [1]. For this solution, the 3 dimensional space begins singular and decelerates as the universe evolves.

If the number of extra dimensions exceeds 1 , then there exists another interesting branch of solutions. For this other branch, the $n>1$ contracting dimensions drive the 3 spatial dimensions not only to expand, but what is more, to inflate.

There is no cosmological constant nor potential driving the inflation. Thus higher dimensional theories exhibit an example of gravity-driven, kinetic inflation [2], [3]. The possibility of kinetic inflation, in the absence of all conventional potential sources, was first discussed in the context of dynamical Planck mass theories. It is simple to confirm that upon dimensional reduction the higher dimensional theory is in fact equivalent to a 4-dimensional theory with a variable Planck mass.

The superstring dilaton also demonstrates this property of dynamical Planck fields. A kinetic inflation from the dilaton of superstring theories was discussed independently in Refs. [4] and [5]. Recently, there have been interesting studies of an inflating superstring 
cosmology in higher dimensions (see for example [6], [7]). In those papers it is the dilaton which drives the inflation. The extra dimensions exist only as a consequence of the nature of string theory. By contrast, in this paper it is the extra dimensions themselves which drive the inflation. As will be discussed, the extra dimensions act very much like the superstring dilaton.

While it is possible to drive the three dimensional world to inflate, a successful completion of kinetic inflation has yet to be found. For the particular higher dimensional model discussed in this paper, there will be a graceful exit problem, as there is for the superstring cosmology [8]. For a discussion of the general obstacle facing the simplest dynamical Planck mass models see Ref. [9].

In the presence of matter sources, Kaluza-Klein models [10] are already known to show inflationary behavior. It was discovered firstly in a radiation dominated universe [11]. The focus of Refs. [11] was on the effective radiation entropy increase. Even if the net $N$-dimensional entropy is conserved, the effective 3-dimensional entropy can increase. In essence, the contracting dimensions squeeze entropy into the expanding dimensions. Unfortunately, unless there is some tuning of parameters, the increase in entropy is too small to solve the cosmological problems. Since these original papers, many source terms have been exploited to fuel inflation in a universe with extra dimensions. Anti-symmetric tensor fields predicted from supergravity [12], stringy fluids [13], as well as curvatures have all been considered as sources. [

The difference here is that there are no matter sources at all. The solutions presented in this paper are strictly vacuum solutions. There exists only the locally flat spacetime itself. By stripping the universe down to essentials, a connection can be made with the dilaton cosmologies mentioned above.

\footnotetext{
${ }^{1}$ Since the writing of this paper, I have discovered reference [19 which considers dust in a higherdimensional universe. The inflationary solution can be recovered as limiting case of [19].
} 
In addition to the familiar 3 space and 1 time dimensions, consider the addition of $n$ extra spacelike dimensions. Let the 3 -volume expands while the $n$-volume contracts. It is an initial condition choice to begin a universe with all spatial dimensions expanding, all contracting, or a few of each. The naturalness of these initial choices will not be defended in this paper. A natural mechanism to compactify additional dimensions has long been sought in theories which involve higher dimensions. If such a mechanism exists, then the anisotropy of the spacetime is not a special initial condition but instead is generated dynamically. While the mechanics of a realistic model will likely be more complicated than those of the simple model studied here, it seems fair to conjecture that the basic features will be generic.

Take the action to be the Einstein theory of gravity generalized to higher dimensions:

$$
A=\int d^{N} x \sqrt{-g^{(N)}}\left[\frac{M_{N}^{2}}{16 \pi} \mathcal{R}^{(N)}\right],
$$

where the total dimension of the spacetime is $N=n+4$. The $N$-dimensional Planck mass is $M_{N}$. The assumption is made that the metric divides simply into an $N$-dimensional homogeneous but anisotropic metric. Three spacelike dimensions are taken to expand isotropically while the other $n$ spacelike dimensions are taken to contract isotropically. The metric is of the form

$$
d s_{(N)}^{2}=-d t^{2}+a^{2}(t) h_{i j}+b^{2}(t) k_{m n}
$$

The indices $i, j$ run from $1 . .3$ and the indices $m, n$ run from $4 . . n+3$. The scale factor of the 3 -space is $a(t)$ and that of the $n$-space is $b(t)$.

The vacuum Einstein equations lead to the following set of equations:

$$
\begin{aligned}
3 \frac{\ddot{a}}{a}+n \frac{\ddot{b}}{b} & =0 \\
\frac{\ddot{a}}{a}+\left(2 H_{a}+n H_{b}\right) H_{a}+\frac{2 \kappa^{(3)}}{a^{2}} & =0 \\
\frac{\ddot{b}}{b}+\left(3 H_{a}+(n-1) H_{b}\right) H_{b}+\frac{(n-1) \kappa^{(n)}}{b^{2}} & =0,
\end{aligned}
$$

where 


$$
\begin{aligned}
H_{a} & \equiv \frac{\dot{a}}{a} \\
H_{b} & \equiv \frac{\dot{b}}{b}
\end{aligned}
$$

and the local curvatures of the internal dimensions, $\kappa^{(3)}$ and $\kappa^{(n)}$, can assume the values $0,1,-1$. For the remainder of this paper all spatial dimensions are taken to be locally flat so that $\kappa^{(3)}=\kappa^{(n)}=0$. The manifold could still be closed as is the case with a locally flat toroid.

The set of equations (1.3)-(1.5) can be manipulated into the set of equations,

$$
\begin{aligned}
H_{a}^{2}+n H_{a} H_{b}+\frac{n(n-1)}{6} H_{b}^{2} & =0 \\
\dot{H}_{a}+\left(3 H_{a}+n H_{b}\right) H_{a} & =0 \\
\dot{H}_{b}+\left(3 H_{a}+n H_{b}\right) H_{b} & =0 .
\end{aligned}
$$

These equations can be solved very straightforwardly. It is possible that both $H_{a}=H_{b}=0$. In that case, the universe is empty and flat and nothing happens. Alternatively we can assume that neither $H_{a}$ nor $H_{b}$ vanishes.

Firstly, the constraint equation (1.8) can be solved 2 for $H_{b} / H_{a}$,

$$
\frac{H_{b}}{H_{a}}=-\left(\frac{3 n \pm \sqrt{3 n^{2}+6 n}}{n(n-1)}\right)
$$

with $n>1$. For $n=1$ there is only one solution to (1.8), $H_{b}=-H_{a}$. If instead $n>1$, there are two roots to this equation. The lower sign corresponds to the decelerating solution found 14 years ago [1] which has since become a canonical reference (see for instance [15], [16]). The upper sign on the other hand corresponds to an inflationary epoch of the 3-space.

It is simple to see that one of the roots corresponds to an accelerated expansion while the other corresponds to a decelerated expansion. From eqn (1.11) notice that for both roots

\footnotetext{
${ }^{2}$ Independently, A. Coley has also found this to be the most general vacuum solution, though the inflationary behavior was not identified [14].
} 
the ratio $H_{a} / H_{b}<0$. Thus if the $n$ extra dimensions contract, the 3 -space expands. Using (1.11) in $(1.9)$ gives

$$
\frac{\dot{H}_{a}}{H_{a}^{2}}=\left(\frac{3 \pm \sqrt{3 n^{2}+6 n}}{(n-1)}\right)
$$

If the upper sign is operative, eqn (1.12) shows that $\dot{H}_{a}>0$. The Hubble expansion gets ever faster and the scale factor $a$ is accelerated. If instead the lower sign is operative, then $\dot{H}_{a}<0$ for all $n>1$ and the expansion gets ever slower. Since $\ddot{a} / a=\dot{H}_{a}+H_{a}^{2}$, it follows from (1.12) that

$$
\frac{\ddot{a}}{a}=\left(\frac{n+2 \pm \sqrt{3 n^{2}+6 n}}{(n-1)}\right) H_{a}^{2} .
$$

When the lower sign solution applies, $\ddot{a}$ is negative and the scale factor decelerates.

To distinguish the two possibilities, the cosmologies will be solved separately for the two roots. For completeness, the decelerating solution of [1] will be reproduced here.

Integrating (1.12) over $d t$ with the lower sign gives

$$
\frac{-1}{H_{a}(t)}+\frac{1}{H_{a}\left(t_{b}\right)}=\left(\frac{3-\sqrt{3 n^{2}+6 n}}{(n-1)}\right) \Delta t,
$$

where $H_{a}\left(t_{b}\right)$ is a constant of integration and $\Delta t=t-t_{b}$. For simplicity $t_{b}$ will hereafter be taken as $t_{b}=0$. From eqn (1.12) we know that $H_{a}$ decreases with time for the lower branch solution. Consequently, $1 / H_{a}(0)<1 / H_{a}(t)$ for $t>0$. An initial condition choice can then be made that $1 / H_{a}(0)=0$. With these specifications eqn (1.14) can be rearranged and integrated to give

$$
a=\bar{a}\left(\frac{t}{\bar{t}}\right)^{r}
$$

where $\bar{a} / \bar{t}^{r}$ is a constant of integration and

$$
r=\frac{3+\sqrt{3 n^{2}+6 n}}{3(n+3)} .
$$

The exponent $r$ is always less than 1, which confirms the previous argument that the expansion of the 3 -space decelerates. From eqn (1.11), $b(t)$ is found to be 


$$
b=\bar{b}\left(\frac{t}{\bar{t}}\right)^{q}
$$

where $\bar{b}$ is a constant of integration and

$$
q=\frac{n-\sqrt{3 n^{2}+6 n}}{n(n+3)} .
$$

This solution gives a generalized Kasner metric [17]

$$
d s^{2}=-d t^{2}+\bar{a}\left(\frac{t}{\bar{t}}\right)^{2 r} d x_{(3)}^{2}+\bar{b}\left(\frac{t}{\bar{t}}\right)^{2 q} d x_{(n)}^{2} .
$$

The exponents obey the required relations

$$
\begin{gathered}
3 r+n q=1 \\
3 r^{2}+n q^{2}=1
\end{gathered}
$$

This is precisely the cosmology found by Chodos and Detweiler in 1980 [1]. The decelerating solution is quickly generalized to $d$ isotropically expanding dimensions and $n$ isotropically contracting dimensions with

$$
\begin{aligned}
& r \rightarrow \frac{d+\sqrt{n d(n+d-1)}}{d(n+d)} \\
& q \rightarrow \frac{n-\sqrt{n d(n+d-1)}}{n(n+d)}
\end{aligned}
$$

Now for the accelerating root. Eqn (1.12) can again be integrated over $d t$, this time for the upper sign solution,

$$
\frac{-1}{H_{a}(t)}+\frac{1}{H_{a}\left(t_{b}\right)}=\left(\frac{3+\sqrt{3 n^{2}+6 n}}{(n-1)}\right) \Delta t,
$$

where again $H_{a}\left(t_{b}\right)$ is an integration constant, $\Delta t=t-t_{b}$, and $t_{b}$ is taken hereafter as zero. From eqn (1.12) it was argued that the Hubble expansion for the 3 dimensions grew with time. As a result, $1 / H_{a}(0)>1 / H_{a}(t)$ for $t>0$. This constant of integration cannot therefore be ignored. Retaining $H_{a}(0)$, eqn (1.24) can be solved for $H_{a}(t)$,

$$
H_{a}(t)=\frac{H_{a}(0)}{[1-t / \bar{t}]}
$$

where the constant $\bar{t}$ is defined by

$$
\left(\frac{1}{\bar{t}}\right)=\left(\frac{3+\sqrt{3 n^{2}+6 n}}{(n-1)}\right) H_{a}(0) .
$$




\section{FIGURES}

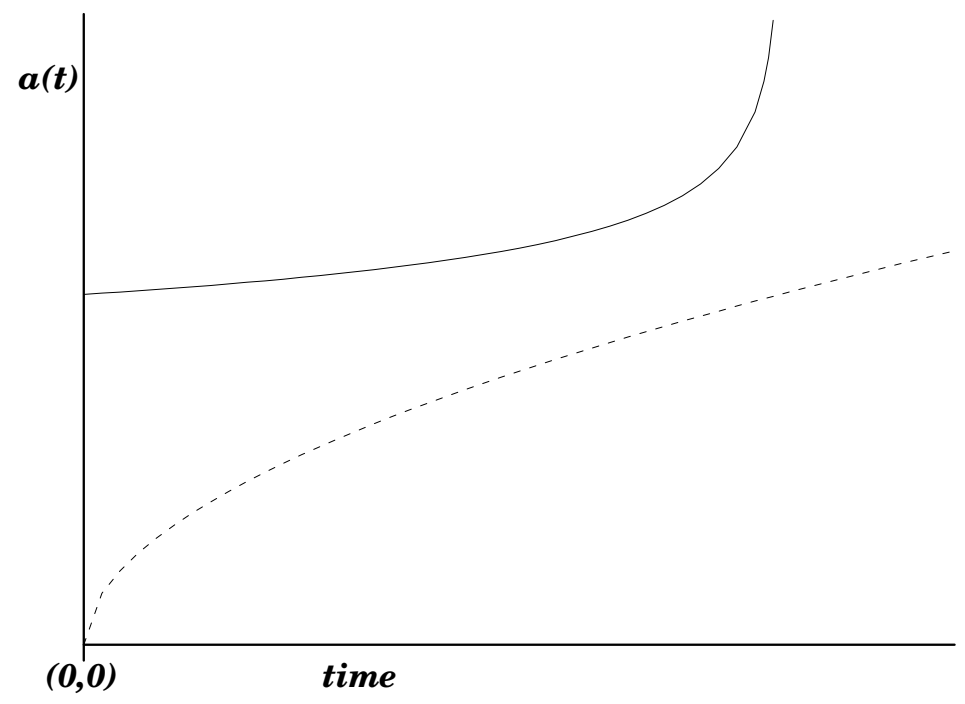

FIG. 1. The scale factor $a(t)$ of the 3 -space for both the accelerating solution (solid line) and the decelerating solution (dashed line). The time axis is drawn from $t=0$ to just after $t=\bar{t}$.

Eqn (1.25) can be integrated over $d t$ to solve for $a(t)$,

$$
a=\frac{\bar{a}}{[1-t / \bar{t}]^{p}}
$$

with $\bar{a}$ a constant of integration and the exponent $p$ defined by

$$
p \equiv \frac{-3+\sqrt{3 n^{2}+6 n}}{3(n+3)} \text {. }
$$

For $n>1, p>0$. The acceleration can be computed directly from (1.27),

$$
\frac{\ddot{a}}{a}=\left(\frac{p}{\bar{t}}\right) \frac{p+1}{\bar{t}} \frac{1}{[1-t / \bar{t}]^{2}}>0
$$

This verifies that the 3 -volume does grow at an accelerated rate; that is, it inflates. Figure 1 sketches the behavior of the scale factor $a(t)$ with time for both the accelerating solution of (1.27) and the decelerating solution of (1.15).

As before, eqn (1.11) leads to the solution for $b(t)$ 


$$
b(t)=\bar{b}[1-t / \bar{t}]^{w}
$$

where $\bar{b}$ is a constant of integration and

$$
w \equiv \frac{n+\sqrt{3 n^{2}+6 n}}{n(n+3)} .
$$

Figure 2, illustrates the behavior of the $n$-space scale factor with time for both the accelerating solution of (1.30) and the decelerating solution of (1.17).

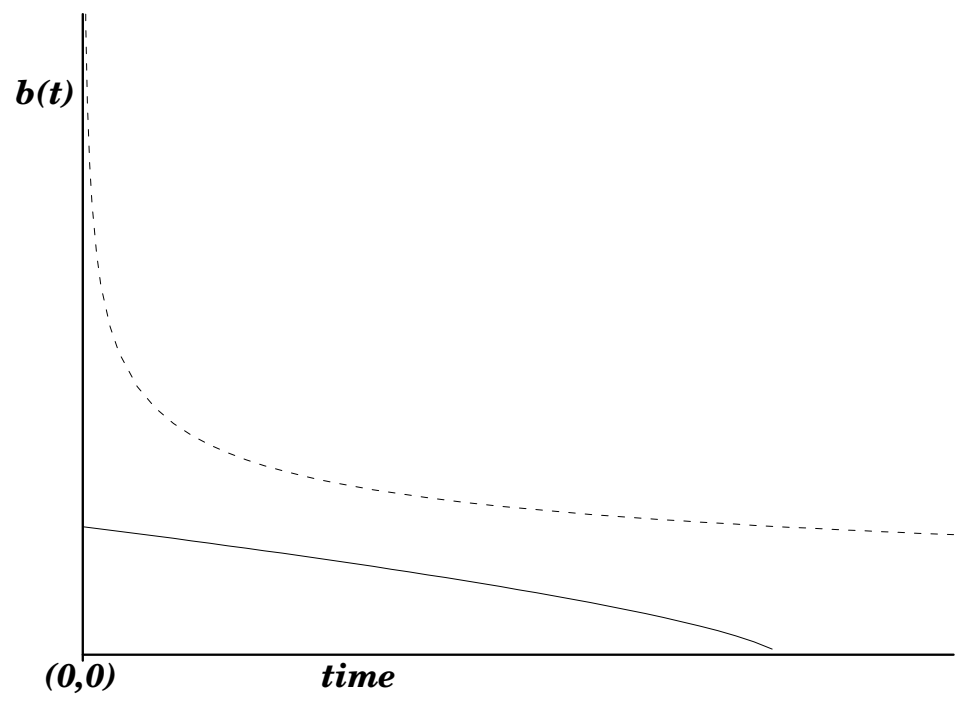

FIG. 2. The scale factor $b(t)$ of the $n$-space for both the accelerating solution (solid line) and the decelerating solution (dashed line). The time axis is again drawn from $t=0$ to just after $t=\bar{t}$.

This branch gives a generalized Kasner metric of the form,

$$
d s^{2}=-d t^{2}+\bar{a}\left(1-\frac{t}{\bar{t}}\right)^{-2 p} d x_{(3)}^{2}+\bar{b}\left(1-\frac{t}{\bar{t}}\right)^{2 w} d x_{(n)}^{2}
$$

The exponents again obey the required relations

$$
\begin{aligned}
& -3 p+n w=1 \\
& 3 p^{2}+n w^{2}=1 .
\end{aligned}
$$


The accelerating solution can be simply generalized to $d$ isotropically expanding dimensions and $n$ isotropically contracting dimensions with

$$
\begin{gathered}
p \rightarrow \frac{-d+\sqrt{n d(n+d-1)}}{d(n+d)} \\
w \rightarrow \frac{n+\sqrt{n d(n+d-1)}}{n(n+d)} .
\end{gathered}
$$

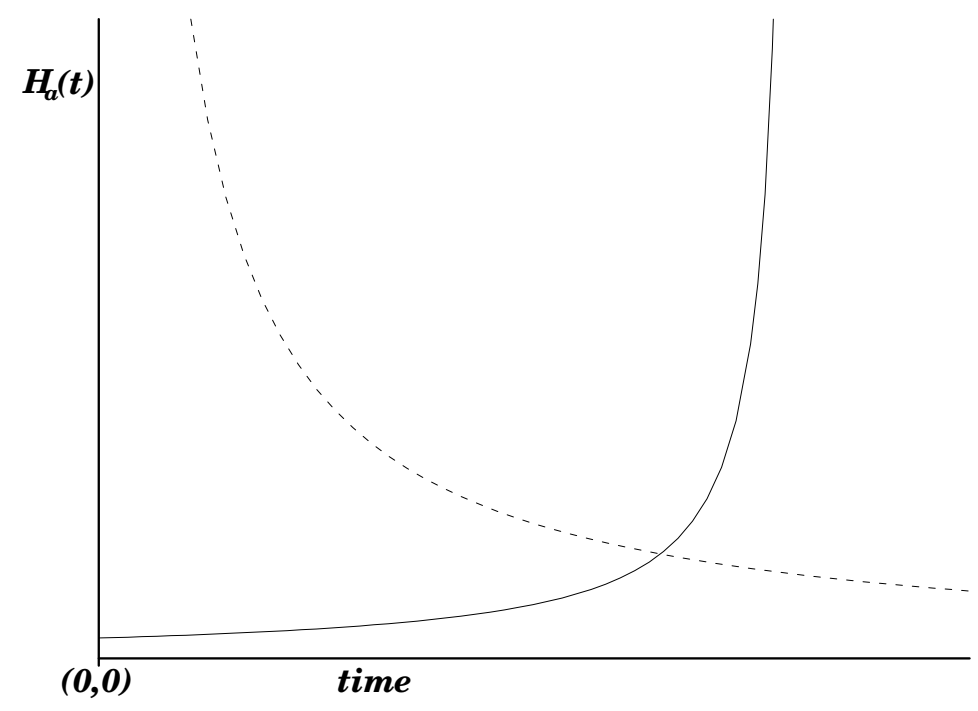

FIG. 3. The Hubble expansion of the 3 -volume, $H_{a}(t)$ for both the accelerating solution (solid line) and the decelerating solution (dashed line).

At time $t=t_{b}=0$, the two scale factors are a finite size, $a=\bar{a}$ and $b=\bar{b}$. The universe appears to begin nonsingular as $a$ is driven to zero and $b$ is driven to $\infty$ only in the infinite past, $t=-\infty$. The expansion of the 3-space gets ever faster as time moves forward. As the $n$-space contracts, the 3 -space inflates. The universe is ultimately ushered into a future singularity. At finite time $t=\bar{t}, a \rightarrow \infty$ while $b \rightarrow 0$. Also, the Hubble expansions show the singular behavior; $H_{a}(\bar{t}) \rightarrow \infty$ while $H_{b}(\bar{t}) \rightarrow-\infty$ (see figures 3 and 4 ). More formally, the spacetime is geodesically incomplete as geodesics will end in finite affine parameter. 


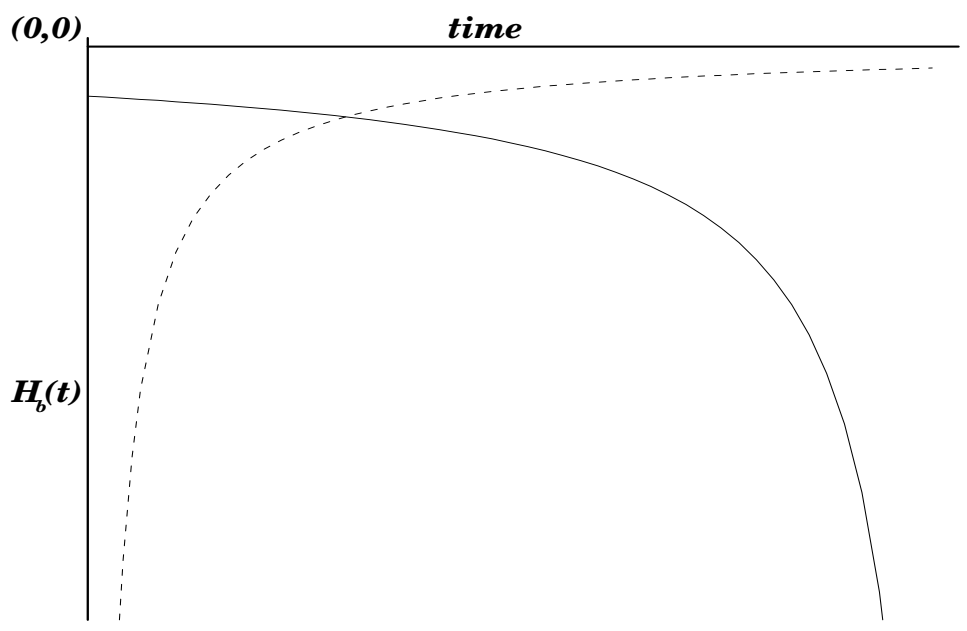

FIG. 4. The Hubble contraction of the $n$-volume, $H_{b}(t)$ for both the accelerating solution (solid line) and the decelerating solution (dashed line).

It is well known that upon dimensional reduction, the radius of the extra dimensions acts just as a dynamical Planck mass [10]. Thus the higher dimensional theory can be related to a generalized Jordan-Brans-Dicke (JBD) theory [18]. Dynamical Planck fields are often generated in particle theories, either through simple quantum corrections or through the full machinery of theories such as superstrings. In a higher dimensional universe, the dynamical Planck mass has a geometric interpretation. It is related to the radius of the compact internal dimensions. To my mind, a geometric source for the dynamical Planck mass is more aesthetic than a particle theory source. It reinforces the suggestion that the entire scenario is purely and truly gravity driven.

Begin again with the action for Einstein gravity generalized to $N=n+4$ dimensions,

$$
A=\int d^{N} x \sqrt{-g^{(N)}}\left[\frac{M_{N}^{2}}{16 \pi} \mathcal{R}^{(N)}\right] .
$$

As is often done, the action can be reduced to a four-dimensional theory by integrating over the extra dimensions, 


$$
S=\int d^{4} x \sqrt{-g^{(4)}} \int d^{n} x b^{n} \frac{M_{N}^{2}}{16 \pi}\left[\mathcal{R}^{(4)}+n(n-1) \frac{\partial_{\mu} b \partial^{\mu} b}{b^{2}}\right]
$$

The action (1.38) is equivalent to a 4-dimensional JBD theory of a dynamical Planck mass,

$$
\int d^{4} x \sqrt{-g^{(4)}}\left[\frac{\Phi}{16 \pi} \mathcal{R}^{(4)}-\omega(\Phi) \frac{\partial_{\mu} \Phi \partial^{\mu} \Phi}{16 \pi \Phi}\right]
$$

with the definitions

$$
\Phi \equiv\left(\int d^{n} x\right) M_{N}^{2} b^{n}
$$

and

$$
\omega \equiv-1+\frac{1}{n}
$$

The kinetic coupling parameter $\omega$ is a negative constant for $n>1$. Notice, there is no potential nor cosmological constant in the dimensionally reduced action (1.39).

The N-dimensional accelerating solution is thus identical to an example of the kinetic driven acceleration from a dynamical Planck mass in $(3+1)$-dimensions. In references [2] and [3] it was shown that the peculiar kinetic energy of a dynamical Planck mass could drive the scale factor of the universe to accelerate. Large families of such theories are possible and can be identified by a bound on the kinetic couping parameter $\omega(\Phi)$. For a vacuum solution, the bound on $\omega(\Phi)$ is

$$
(1+2 \omega / 3)^{1 / 2} \mp 1-\frac{1}{3(1+2 \omega / 3)^{3 / 2}} \frac{\partial \omega(\Phi)}{\partial \ln \Phi}<0
$$

[3]. If $\omega$ is a negative constant, then only for the branch of solutions corresponding to the upper sign and a decreasing Planck mass will the universe expand at an accelerated rate. In the example of this paper, the effective $\omega$ is a negative constant, the branch of solutions corresponds to the required branch, and the decrease in the Planck mass is effected by the decrease in the scale of the extra dimensions.

The dilaton of superstring theories in 4-D can also provide an accelerating branch of solutions. The superstring dilaton is akin to a JBD theory with $\omega=-1$. The dimensionally 
reduced theory of this paper, giving a JBD theory with $\omega=-1+1 / n$, is thus very similar to the superstring dilaton. Unfortunately, it was found in the string case that inflation could not be completed with success [8] [9]. To be specific, our universe today does not connect smoothly onto the branch of solutions which corresponds to inflation. A mechanism to induce a branch change and provide a graceful exit seems thus far to have eluded the string cosmology [8]. The graceful exit problem which challenges superstring inflation will challenge higher-dimensional inflation as well.

The graceless exit can be demonstrated simply by inverting eqn (1.11) to find $H_{a}$ for the inflationary branch of solutions,

$$
H_{a}=-\frac{n}{2} H_{b}-\sqrt{\frac{\left(n^{2}+2 n\right)}{12} H_{b}^{2}+\frac{8 \pi}{3 M_{N}^{2}} \rho} .
$$

In the above expression, possible contributions from matter sources have been included in the energy density $\rho$. Today's universe evolves roughly as

$$
H_{a, o} \sim+\sqrt{\frac{8 \pi G_{o}}{3} \rho_{o}} .
$$

The standard Einstein equations allow two branches, one expanding and one contracting. The expanding branch is chosen as the physically relevant one. Clearly the inflationary solution of (1.43) is on the wrong branch. Even if a mechanism existed to stabilize the internal dimensions so that $H_{b} \rightarrow 0$ and matter domination took over, it is clear from (1.43) that the 3 -volume would ultimately contract, $H_{a} \rightarrow-\sqrt{8 \pi \rho / 3 M_{N}^{2}}$. A branch change is needed if the inflationary solution is to connect smoothly onto our expanding cosmology. 3

Regardless of its ultimate fate as an inflationary model, the higher-dimensional theory exhibits another interesting feature. The universe begins cold and empty. With just a kick as an initial condition, the cosmology evolves into a future singularity from a completely regular state. This mild and quiet universe, perhaps a simple thing to create, evolves into an era of

\footnotetext{
${ }^{3}$ Even with source terms driving inflation, as in Refs. [11- 13], such a branch change may be needed in some cases.
} 
quantum gravity. Thus a possible explanation is offered as to why the early universe was so fiercely energetic. The cold beginning remains a fascinating alternative to the standard hot big bang lore.

\section{Acknowledgements}

Thank you to J.R. Bond, A. Coley, N.J. Cornish and G. Starkman for their thoughts on this project. I am also grateful for the additional support of the Jeffrey L. Bishop Fellowship. 


\section{REFERENCES}

[1] A. Chodos and S. Detweiler, Phys. Rev. D 21, 2176 (1980).

[2] K. Freese and J.J. Levin, preprint UM-AC-93-23, (1993);

[3] J.J. Levin, to be published in Phys. Rev. D, preprint CITA-94-18, gr-qc/9405061.

[4] G. Veneziano, Phys. Lett. B 265, 287 (1991).

[5] M. Gasperini and G. Veneziano, Astropart. Phys I, 317 (1993).

[6] E. J. Copeland, A. Lahiri, and D. Wands, preprint SUSX-TH-94/3-7, SUSSEX-AST94/6-2, hep-th/94060216 (1994).

[7] K. Behrndt and S. Förste, preprint DESY 933-118, hep-th/9308131 (1993); K. Behrndt and S. Förste, preprint SLAC-PUB-6471, RI-2-94, hep-th/9403179 (1994).

[8] R. Brustein and G. Veneziano, Phys. Lett. B 329, 429 (1994).

[9] J.J. Levin, to be published in Phys. Rev. D, CITA-94-25, hep-th/9407101.

[10] Th. Kaluza, Sitzungsber, Preuss. Akad. Wiss. Phys. Math. Kl. 966 (1921); O. Klein, Z. Phys. 37, 895 (1929).

[11] D. Sadhev, Phys. Lett. 137 B, 155 (1984); E.W. Kolb, D. Lindley and D. Seckel, Phys. Rev. D 30, 1205 (1984); R.B. Abbott, S.M. Barr and S.D. Ellis Phys. Rev. D 30, 720 (1984).

[12] R.G. Moorhouse and J. Nixon, Nucl. Phys. B261 (1985) 172.

[13] M. Gasperini, N. Sánchez and G. Veneziano, Nucl. Phys B364 (1991) 365.

[14] A. Coley, Astrophys. J. 427, 585 (1994).

[15] Eds. T. Appelquist, A. Chodos, and P.G.O. Freund, Modern Kaluza-Klein Theories, (Menlo Park, CA: Addison-Wesley Publishing Company, Inc.) (1987).

[16] E.W. Kolb and M.S. Turner, The Early Universe, (Redwood City, CA: Addison-Wesley Publishing Company, Inc.) (1990).

[17] E. Kasner, Am. J. Math. 43, 217-221 (1921).

[18] P. Jordan, Z. Phys.157 112 (1959); C. Brans and C. H. Dicke, Phys. Rev. 24, 925 (1961); R.H. Dicke, Ap. J., 152, 1 (1968); P.G. Bergmann, Int. J. Theor. Phys., 125 (1968); R.V.Wagoner, Phys. Rev D 1, 3209 (1970).

[19] H. Ishihara, Prog. Theor. Phys. 72, 376 (1984). 